Studia Psychologica, Vol. 63, No. 1, 2021, 77-93

https://doi.org/10.31577/sp.2021.01.815

\title{
The Brief Partner Behavioral Control Scale (B-PBCS): Development and Validation
}

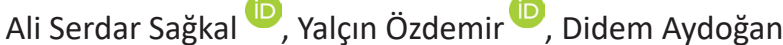 \\ Aydın Adnan Menderes University, Faculty of Education, Department of Psychological Counseling and Guidance, Central \\ campus, 09100 Aydın, Turkey
}

\begin{abstract}
Despite the large growth of close relationships literature, partner behavioral control has been rarely the primary focus of research. It is also evident that the instruments for measuring partner behavioral control are scarce. Thus, the present study aims to develop and validate a 9-item Brief Partner Behavioral Control Scale (B-PBCS). The validity and reliability of the B-PBCS were examined in a sample of emerging adults $\left(N=511 ; 81.8 \%\right.$ females; Range $\left._{\text {age }}=18-25\right)$ who are currently in a premarital romantic relationship. To assess psychometric properties of the B-PBCS, we conducted a series of tests examining factor structure (exploratory and confirmatory factor analyses), measurement invariance (configural and metric invariance), reliability (internal consistency and test-retest reliability), and predictive validity. Results demonstrated that the B-PBCS has a two-factor solution (overt and covert partner behavioral control), acceptable measurement invariance, internal consistency, test-retest reliability, and predictive validity. The overt partner behavioral control at baseline assessment has been linked to romantic relationship conflict and psychological distress at 14-week follow-up assessment even after controlling for sex, age, and relationship duration. In conclusion, the B-PBCS offers a valid and brief measure for assessing partner behavioral control.
\end{abstract}

Key words: emerging adults, partner behavioral control, premarital romantic relationships, scale development, validation

\section{Introduction}

Over the last two decades, a large body of research has focused on understanding the nature and significance of romantic relation- ships in emerging adulthood. As a distinct period from adolescence and young adulthood, emerging adulthood, the period from the late teens through the late twenties, is characterized as the age of identity explorations, instability, self-focus, feeling in-between, and

This study was presented as an oral presentation at the International Association Relationship Research (IARR) Mini-Conference 2019, July 18-21, Brighton, UK.

Correspondence concerning this article should be addressed to Ali Serdar Sağkal, Aydın Adnan Menderes University, Faculty of Education, Department of Psychological Counseling and Guidance, Central Campus, 09100, Aydın, Turkey. E-mail: aliserdarsagkal@gmail.com 
possibilities (Arnett, 2000). In the $21^{\text {st }}$ century industrialized countries, with changing societal trends and expectations, marriage rates tend to decrease and the mean age at first marriage tends to increase gradually (Eurostat, 2019). Instead of committing to adult roles and responsibilities, today's youth spend more time in romance and love before settling on permanent relationships (Arnett, 2000, 2004, 2007). Therefore, forming, maintaining, and ending romantic relationships appears as a critical developmental task in emerging adults' lives (Snyder, 2006). Achievement of this developmental task is believed to satisfy intimacy needs (Fincham \& Cui, 2011), contribute to finding a meaningful life (Mayseless \& Keren, 2014), and associate with progress in adopting adult roles and responsibilities (Barry, Madsen, Nelson, Carroll, \& Badger, 2009).

Theoretical perspectives (La Guardia \& Patrick, 2008) and empirical findings (e.g., Dush \& Amato, 2005; Patrick, Knee, Canevello, \& Lonsbary, 2007; Sağkal \& Özdemir, 2020) highlight that there is a significant link between romantic relationship functioning and individual well-being. Although sustaining a healthy romantic relationship importantly contributes to relational and individual processes, it is also evident that negative romantic experiences increase the odds of relationship conflict (Mackinnon et al., 2012), relationship dissolution (Fine \& Harvey, 2006) as well as mental health problems for individuals (DiBello, Preddy, Øverup, \& Neighbors, 2017). In particular, partner behavioral control appears as an important risk factor for psychological adjustment problems and dating violence (e.g., Follingstad, Bradley, Helff, \& Laughlin, 2002; Graham-Kevan \& Archer, 2008; Khaleque, 2012). Previous research has extensively documented the degree to which parental psychological and behavioral control affects children's and adolescents' psycho- social development (e.g., Barber, Olsen, \& Shagle, 1994; Steinberg, Elmen, \& Mounts, 1989; Shek, 2006). However, the impact of perceived partner behavioral control on relational and individual outcomes has been rarely addressed in close relationships literature (e.g., Basha, 2014; Rohner \& Khaleque, 2008; Stark, 2007).

\section{Partner Behavioral Control and Its Expanded Operationalization}

Partner behavioral control has already been conceptualized as the demands, directives, proscriptions, and prescriptions that a partner places on his/her intimate partner's behaviors (Rohner, 2005a, 2005b). Partner behavioral control refers to the quality of relationship from a permissive structure to enforcing a strict limit for restrictions on partner's behaviors. However, in our conceptualization, partner behavioral control involves not only overt (i.e., trying to change partner's behaviors) but also covert behavioral control (i.e., attempts to know what a partner does on a daily routine). We operationalize the overt partner behavioral control as limiting the partner's behaviors in a way that can be perceived by the romantic partner. For example, trying to change the decisions and behaviors of the partner is a prominent indicator of overt partner behavioral control. As a second dimension, covert partner behavioral control reflects control behaviors, which are undetected by the partner but still result in restrictions and limitations. For instance, attempts of a partner to demand from the other where he or she spends time, with whom and what does $s /$ he do during the day would be presented as indicators of covert partner behavioral control. Furthermore, covert partner behavioral control, as a closely related construct to the partner monitoring, includes tracking the daily behaviors of the partner. In 
covert partner behavioral control, by monitoring daily activities of the partner, one tries to create a rule over his or her romantic partner. However, to date, partner monitoring has been frequently addressed in social networking site research (e.g., Belu, Lee, \& Sullivan, 2016; Fox \& Tokunaga, 2015; Van Ouytsel, Walrave, Ponnet, Willems, \& Van Dam, 2019) and we still do not have strong evidence regarding the effects of partner monitoring in real life settings. Therefore, based on broader conceptualization of partner behavioral control, it seems important to fill the gap and develop a new valid and reliable instrument incorporating these two dimensions in the assessment of partner behavioral control. Furthermore, it is also crucial that assessing how overt and covert partner behavioral control differentially predicts individual and relational outcomes in couples would further advance close relationships literature.

\section{Existing Partner Behavioral Control Measures and Empirical Findings}

Researchers usually examine partner behavioral control either on the basis of Interpersonal Acceptance-Rejection Theory (IPARTheory; Rohner, 2014) or on the basis of Intimate Partner Violence and Abuse (IPVA; Fincham, Cui, Braithwaite, \& Pasley, 2008). The IPARTheory, as an evidence-based theory of socialization and life span development, attempts to understand the antecedents and outcomes of interpersonal acceptance/rejection and control (Rohner, 2014, 2016). The IPARTheory suggests that the degree of acceptance, rejection, and control experienced in interpersonal relationships is significantly and panculturally related to individuals' personality dispositions and psychological adjustment (Rohner, 2016). Parental Acceptance-Rejection/Control Questionnaire (PARQ/Control; Rohner, 2005a) and Intimate Partner Acceptance-Rejection/Con- trol Questionnaire (IPAR/CQ; Rohner, 2005b) are two main self-report questionnaires used widely in IPARTheory research. While PARQ/ Control assesses individuals' perceptions of maternal and paternal accepting-rejecting and controlling behaviors, IPAR/CQ measures the same dimensions with reference to intimate partners. The results of an overview of 12 meta-analyses summarizing results from 31 countries and 149440 participants (Khaleque $\&$ Ali, 2017) support the basic postulates of IPARTheory and confirm the cross-cultural robustness of the scales used in IPARTheory research. However, it must be noted that existing studies (e.g., Geitsidou \& Giovazolias, 2016; Khaleque, Shirin, \& Uddin, 2013; Malik \& Rohner, 2016; Rohner, Melendez, \& Kraimer-Rickaby, 2008) almost exclusively have focused on the warmth (acceptance-rejection) dimension of PARQ/Control and IPAR/CQ. There are comparatively fewer studies (e.g., Khaleque, 2012) assessing the effects of intimate partner behavioral control on individuals' psychological and relational outcomes. It is also evident that although data have been collected with regard to control dimension of $\mathrm{PARQ} /$ Control and/or IPAR/CQ, researchers usually did not use this dimension in the subsequent analyses (e.g., Khaleque \& Rohner, 2013; Khaleque, Rohner, \& Laukkala, 2008; Malik \& Rohner, 2016). Nevertheless, limited empirical evidence (e.g., Khaleque, 2012) indicates that there is a significant negative link between perceived intimate partner behavioral control and psychological adjustment.

Furthermore, controlling behaviors perpetrated in romantic relationships are also addressed in the intimate partner violence and abuse (IPVA) research. IPVA includes violent and abusive physical, sexual, and/or psychological behaviors directed toward current or former partners (Fanslow \& Robinson, 2011; Walker, Sleath, \& Tramontano, 2021). Scholars (Arroya, Lundahl, Butters, Vanderloo, \& Wood, 
2017) argue that a desire to maintain power or exert control over a partner usually lies in the root of IPVA. As a serious public health issue, IPVA affects millions of people and occurs across the lifespan (Centers for Disease Control and Prevention, 2018). According to the World Health Organization's (WHO) technical report (Garcia-Moreno et al., 2013), the global prevalence of intimate partner violence (physical and/or sexual IPV) is 30\%. Prevalence estimates are lowest in the high-income region (23.2\%) and highest in South-East Asia (37.7\%) and Eastern Mediterranean (37\%) regions. Actually, recent findings (Lövestad, Löve, Vaez, \& Krantz, 2017) highlight that as a form of IPVA, the prevalence of controlling behaviors is much more common than physical and sexual violence in intimate relationships. The Intimate Partner Violence Attitude Scale-Revised (IPVAS-Revised; Fincham et al., 2008), Revised Conflict Tactics Scale (Straus, Hamby, Boney-McCoy, \& Sugarman, 1996), Revised Controlling Behaviours Scale (CBS-R; Graham-Kevan \& Archer, 2005), and Multidimensional Measure of Emotional Abuse (MMEA; Murphy \& Hoover, 1999) appear to be the most frequently used instruments in the IPVA literature. Nevertheless, it should be stressed that these measures focus mainly on assessing acts of violence and abuse within dating, cohabiting, and/or marital relationships. As controlling behaviors have been recently defined in the context of IPVA, there is limited number of measures assessing such behaviors comprehensively in the intimate relationships (Sleath, Walker, \& Tramontano, 2018). For example, the CBS-R (Graham-Kevan \& Archer, 2005) involves items related to economic, threatening, intimidating, emotional, and isolating behaviors. However, it is seen that the CBS-R assesses controlling behaviors in the context of coercive control.

Taken together, based on previous literature, it is seen that partner behavioral control has been investigated mostly either within the IPARTheory or IPVA literature. While IPARTheory focuses mostly on the effects of partner acceptance and rejection, IPVA literature focuses mainly on assessing acts of assault, threats, humiliation, intimidation or abuse of one partner over other. In addition, it is observed that while partner behavioral control is extensively assessed in the context of coercive partner behaviors in the IPVA literature, in the IPARTheory the partner behavioral control dimension is dropped from further analyses. Therefore, it may be concluded that there is a need for a new and psychometrically robust measure assessing partner behavioral control from a permissive to a strict structure in romantic relationships. Moreover, it appears that there is a need for a broader conceptualization of partner behavioral control in close relationships literature. In our view, partner behavioral control not only includes overt but also covert acts. Developing an expanded measure of partner behavioral control in terms of overt and covert control and assesing how these two dimensions differentially predict relational and individual outcomes would further contribute to existing literature.

\section{Current Study}

Given that previous research has either overlooked the behavioral control dimension or has provided limited number of findings regarding the role of partner behavioral control on relational and individual functioning (Khaleque, 2001; Khaleque et al., 2008; Parmar \& Rohner, 2005; Rohner \& Khaleque, 2008, 2010), the present study aimed to design a new questionnaire (The Brief Partner Behavioral Control Scale; B-PBCS) that may briefly and robustly capture the construct of partner behavioral control. By developing a brief, valid, and reliable instrument that targets the 
controlling behaviors experienced in romantic relationships, this research may expand future studies on close relationships. Understanding antecedents and outcomes of partner behavioral control would be an important step in developing intervention and prevention programs aimed at enhancing relationship functioning of couples.

\section{Method}

\section{Participants and Procedure}

Turkish emerging adults ( $N=511 ; 81.8 \%$ females), who were in a romantic relationship and unmarried took part in the research. We split overall research sample randomly into two subsamples. The first sample consisted of 256 participants (205 females, Range ${ }_{\text {age }}=$ 18-25, $M_{\text {age }}=20.98, S D_{\text {age }}=1.65$ ) and was used for exploratory factor analysis. The relationship duration ranged from 1 month to 120 months (10 years), with a mean length of 26.22 months ( $S D=24.96)$. The second sample consisted of 255 participants ( $213 \mathrm{fe}$ males, Range $_{\text {age }}=18-25, M_{\text {age }}=21, S D_{\text {age }}=1.68$ ) and was used for confirmatory factor analysis. The relationship duration ranged from 1 month to 111 months (almost 9 years), with a mean length of 23.96 months $(S D=20.61)$. Of the total sample who had completed the surveys at baseline assessment (Time 1), 182 who were continuing the relationship with the same partner from Time 1 completed the surveys at the 14-week follow-up assessment (Time 2). Based on this data set, we calculated measurement invariance, test-retest reliability, and predictive validity of the B-PBCS. The data used in the current study was a portion of the Turkish Longitudinal Romantic Relationship Study (TLRRS; Sağkal \& Özdemir, 2020). The participants were recruited from undergraduate education and psychology courses at a public university in western Tur- key during the spring semester of 2018. Prior to paper-and-pencil survey administration, we explained the nature of the research and obtained participants' informed consent. The research protocols were conducted according to the guidelines of Institutional Ethics Board Committee.

\section{Development of the Brief Partner Behavioral Control Scale (B-PBCS)}

The B-PBCS is a brief measure developed to assess perceptions related to partner's behavioral control. Partner behavioral control was conceptualized in two dimensions: (i) overt partner behavioral control and (ii) covert partner behavioral control. While overt partner behavioral control refers to the demands, directives, proscriptions, and prescriptions that a partner directly place on his/her intimate partner's behaviors, covert partner behavioral control refers to forms of behavioral control, which are masked in socially acceptable ways and undetected by the partner. Based on the Interpersonal Acceptance-Rejection Theory (IPARTheory), Intimate Partner Acceptance-Rejection/Control Questionnaire (IPAR/CQ), IPVA literature, IPVA measures as well as the cultural relevance of the items for Turkish emerging adults, we generated a total of nine items that capture facets of partner behavioral control. Items are rated on a three-point Likert scale (1 = Not like him/her, 2 = Somewhat like him/her, and 3 = A lot like him/her) and higher scores from the scale represent restrictive partner behavioral control. Instructions are as follows: "The following questions are about your experiences with your romantic partner. There are no right or wrong answers. Please choose the answer that best describes your relationship." Following item generation, to increase the content validity, three counseling researchers independently examined the rel- 
evance and representativeness of the B-PBCS items. Lastly, we administered the target scale to a small number of participants $(n=30)$ to test comprehensibility and unambiguousness of the items. After revisions and subsequent modifications, the nine-item initial pool was retained for empirical testing.

\section{Instruments}

In order to assess predictive validity of the $B-P B C S$, we examined the links between B-PBCS, conflict in romantic relationship, and psychological distress. Participants who were continuing the relationship with the same partner from baseline assessment (Time 1) completed the surveys at the 14-week follow-up assessment (Time 2).

Conflict in romantic relationship. Conflict in romantic relationship was measured with the conflict properties dimension of the Children's Perception of Interparental Conflict Scale (CPIC; Grych, Seid, \& Fincham, 1992; Ulu \& Fışıloğlu, 2004). Although the original scale aims to measure perceptions regarding interparental conflict, in the present study we reworded the items so that participants responded to items with reference to romantic relationship conflict (e.g., "Even after we stop arguing, we stay mad at each other"). The scale consists of three subscales, namely, frequency, intensity, and resolution. Participants rated items on a 3-point Likert scale ranging from $1=$ True to $3=$ False, with higher scores indicating frequent, intense, and unresolved romantic relationship conflict. Previous research (Sağkal \& Özdemir, 2019) demonstrated that the Turkish version, which assesses conflict in romantic relationship, has good validity and reliability. In the present study, the Cronbach's alpha coefficients were .84, .77, .76, and .90 for frequency, intensity, resolution, and total scale, respectively.

Psychological distress. Psychological distress was measured with the Kessler Psycho- logical Distress Scale (K10; Kessler et al., 2002). $\mathrm{K} 10$ is a unidimensional, 10 -item, self-report instrument designed to assess psychological distress. Items are responded to on a 5-point Likert scale ranging from $1=$ None of the time to $5=$ All of the time, with higher scores indicating greater psychological distress. The validity and reliability of the Turkish version of the K10 has been confirmed in previous studies (Sağkal \& Özdemir, 2017; Sağkal, Özdemir, \& Koruklu, 2018). In the present study, Cronbach's alpha was 94 .

\section{Data Analysis}

Data analyses were conducted using SPSS version 24.0 and AMOS version 24.0. The total sample was randomly split into two subsamples (Sample 1, $n=256$; Sample 2, $n=255$ ) in order to cross-validate the factor structure of the B-PBCS. We first conducted an exploratory factor analysis (EFA) with principal component analysis (PCA) and oblique rotation. Bartlett's test of sphericity and the Kaiser-Meyer-Olkin (KMO) measure were used to assess sampling adequacy of data for the factor analysis of the B-PBCS items. The eigenvalue $>1.0$ criterion and the scree-plot were used to determine the number of factors to be retained in the model (Tabachnick \& Fidell, 2007). Variables with factor loadings $\geq .40$, communality values $\geq .50$, and cross-loadings $\leq .32$ were retained in the analyses (Worthington \& Whittaker, 2006). To verify the two-factor structure identified in the EFA, we next conducted a confirmatory factor analysis using maximum likelihood estimation. The Comparative Fit Index (CFI), the Tucker-Lewis Index (TLI), and the Root Mean Square Error of Approximation (RMSEA) were used to evaluate the goodnessof-fit of the proposed model. The TLI and CFI values above .90 and the RMSEA values below .06 were considered evidence for acceptable fit (Byrne, 2010). Furthermore, to assure 
the two-factor structure associated with the same items and the same meanings across time, we tested configural and metric invariance of the B-PBCS. We performed chi-square difference test $\left(\Delta \chi^{2}\right)$ to evaluate the relative fit of the nested models. For the reliability of the B-PBCS, we computed Cronbach's alpha and 14-week test-retest reliability coefficients. We also investigated the inter-item correlations and corrected item-total correlations of the B-PBCS. Lastly, we assessed predictive validity by investigating the links between partner behavioral control (overt and covert partner behavioral control), romantic relationship conflict, and psychological distress. A latent path model was used to estimate the predictive role of partner behavioral control at Time 1 (baseline assessment) in romantic relationship conflict and psychological distress at Time 2 (14-week follow-up assessment).

\section{Results}

\section{Factor Structure}

Exploratory Factor Analysis (EFA). The first sample (256 participants, Range age $=18-25$, $\left.M_{\text {age }}=20.98, S D_{\text {age }}=1.65\right)$ was used for the exploratory factor analysis. The results of the Kaiser-Meyer-Olkin measure (.84) and the Bartlett's test of sphericity $\left(\chi^{2}(36)=1101.590\right.$, $p<.001)$ indicated that the adequacy of the data set for factor analysis is appropriate. The underlying structure of the B-PBCS was evaluated through a principal component analysis with oblique rotation (direct oblimin). Inspection of the eigenvalues and the scree plot suggested a two-factor structure for the B-PBCS items. The eigenvalues for the first and second factors were 4.097 and 1.852, respectively. The first factor consisted of 5 items that capture the perceptions related to a partner's attempts at controlling his/her partner's daily activities (e.g, My partner wants to know where I am and what I do during the day) and was labeled "covert partner behavioral control". The second factor consisted of 4 items that capture the perceptions concerning a partner's attempts at controlling his/her partner's behaviors (e.g., My partner always tries to change my behaviors) and was labeled "overt partner behavioral control". Factor loadings ranged from .81 to .86 for covert partner behavioral control and from .73 to .80 for overt partner behavioral control. Findings revealed that all the items evidenced strong loadings on their respective factors without cross-loading problems. Communalities were between .50 and .75 (see also Table 1). The two-factor model accounted for $66.10 \%$ of the variance (dimensions of covert and overt partner behavioral control contribute $45.52 \%$ and $20.58 \%$, respectively).

Confirmatory Factor Analysis (CFA). The factor structure of the 9-item B-PBCS was also examined through the application of CFA on the data from the second sample (255 participants, Range $_{\text {age }}=18-25, M_{\text {age }}=21, S D_{\text {age }}=$ 1.68). Maximum likelihood estimation was used to test the two-factor model. The initial CFA results showed that model fit indices, except TLI and RMSEA, were adequate for the two-factor solution: $\chi^{2}(26)=105.970, \chi^{2} / d f=$ 4.076, $p<.001, \mathrm{CFI}=.91, \mathrm{TLI}=.88$, and RM$\mathrm{SEA}=.11[.09, .13]$. The modification indices suggested the addition of an error covariance term between items 1 and 3 ('My partner wants to know who my friends are' and 'My partner wants to know whom I spend time with') to improve model fit. As these two items overlap in content (loading on the same factor) and adding modification substantially improves model fit, we allowed the error variances of items 1 and 3 to correlate. After adding covariance, the reestimated model yielded good fit for the two-factor solution: $\chi^{2}$ $(25)=34.547, \chi^{2} / d f=1.382, p>.05, \mathrm{CFI}=.99$, $\mathrm{TLI}=.98$, and RMSEA $=.04[.00, .07]$. For the 
Table 1 Exploratory factor analysis of the B-PBCS using Principal Component Analysis with Oblique Rotation (The First Sample: $n=256$ )

\begin{tabular}{|c|c|c|c|c|c|}
\hline Item & Factor I & Factor II & $h^{2}$ & $M$ & $S D$ \\
\hline \multicolumn{6}{|l|}{ Covert partner behavioral control } \\
\hline $\begin{array}{l}\text { 3. My partner wants to know whom I spend time } \\
\text { with. }\end{array}$ & .86 & -.01 & .73 & 2.66 & .53 \\
\hline $\begin{array}{l}\text { 8. When I go somewhere or do something, my } \\
\text { partner wants to know that. }\end{array}$ & .86 & .03 & .75 & 2.60 & .59 \\
\hline $\begin{array}{l}\text { 5. My partner wants to know where I am and what } \\
\text { I do during the day. }\end{array}$ & .85 & .04 & .75 & 2.54 & .61 \\
\hline $\begin{array}{l}\text { 9. My partner wants to know what I do in my daily } \\
\text { routine. }\end{array}$ & .85 & .00 & .72 & 2.54 & .59 \\
\hline 1. My partner wants to know who my friends are. & .81 & -.03 & .64 & 2.69 & .53 \\
\hline \multicolumn{6}{|l|}{ Overt partner behavioral control } \\
\hline 4. My partner controls all my behaviors. & .01 & .80 & .64 & 1.50 & .67 \\
\hline 6. My partner decides whom I will [or not] meet. & .09 & .77 & .63 & 1.30 & .50 \\
\hline $\begin{array}{l}\text { 7. My partner decides how I dress in a given } \\
\text { context. }\end{array}$ & .11 & .74 & .60 & 1.33 & .57 \\
\hline 2. My partner always tries to change my behaviors. & -.13 & .73 & .50 & 1.39 & .60 \\
\hline Eigenvalue & 4.097 & 1.852 & & & \\
\hline Variance explained & $45.52 \%$ & $20.58 \%$ & & & \\
\hline
\end{tabular}

Note. B-BPCS = Brief Partner Behavioral Control Scale; Factor I = covert partner behavioral control; Factor II = overt partner behavioral control; $h^{2}=$ communalities.

9-item B-PBCS, standardized factor loadings ranged from .52 to .84 for covert partner behavioral control and from .59 to .79 for overt partner behavioral control (see Figure 1). All the $t$ values were significantly different from zero at the .001 level. The factor correlation was .42 and significant at the $p<.001$ level.

\section{Measurement Invariance}

We also investigated measurement invariance of the B-PBCS across time using a multi-group analysis in AMOS 24.0. Time 1 and 2 data were used for the analysis of measurement invariance. Configural invariance allowing factor loadings to be freely estimated across time yielded a good fit to the data: $\chi^{2}(50)=$ 65.768, $p>.05, \chi^{2} / d f=1.32, \mathrm{CFI}=.99, \mathrm{TLI}=$
.99 , and RMSEA $=.03 \mathrm{Cl}[.00, .05]$. After establishing configural invariance, we examined the metric invariance of the B-PBCS. Metric invariance constraining factor loadings to equal at Times 1 and 2 resulted in a good fit to the data: $\chi^{2}(59)=76.374, p>.05, \chi^{2} / d f=1.29$, $\mathrm{CFI}=.99, \mathrm{TLI}=.99$, and RMSEA $=.03 \mathrm{Cl}[.00$, .05]. As the chi-square difference test was not statistically significant $\left(\Delta \chi^{2}(9)=10.6, p=.30\right)$, the findings suggested that the two-factor structure of the B-PBCS is invariant over time.

\section{Reliability}

Internal consistency reliability. The internal consistency reliability for the B-PBCS was calculated for both subscales and total scale in each sample. The Cronbach's alpha values 


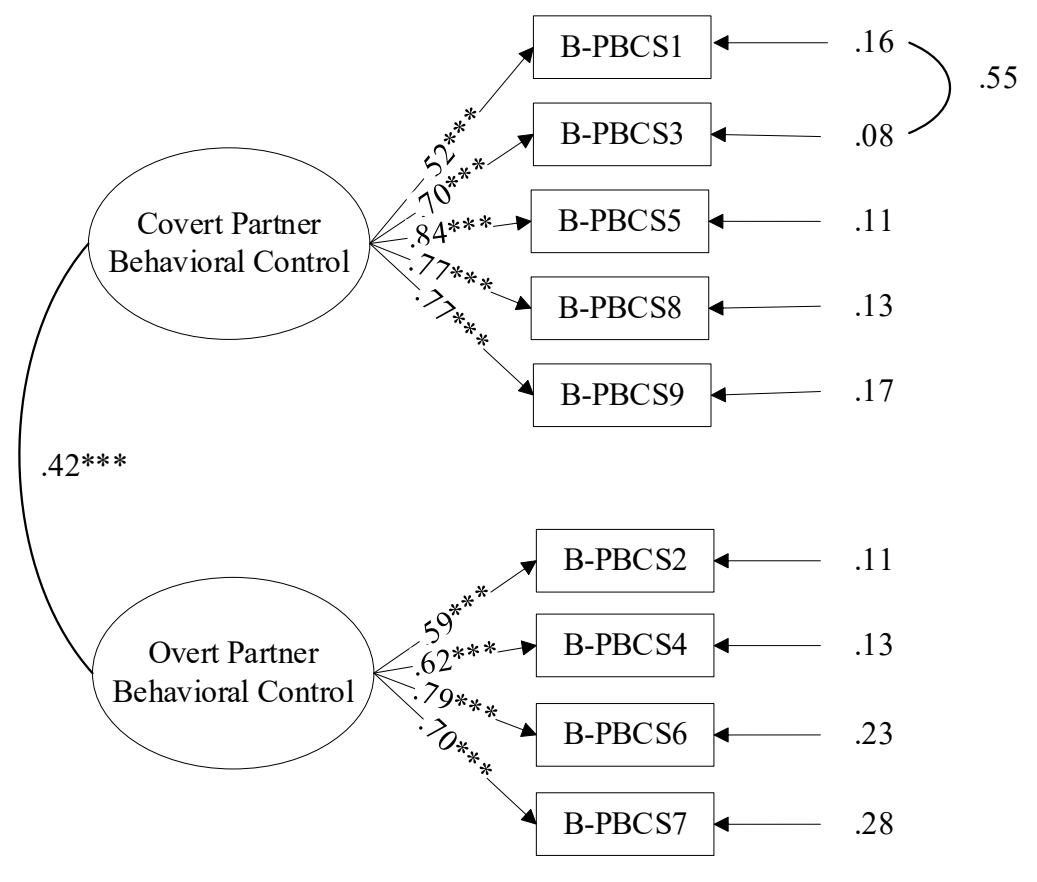

Figure 1 Standardized solution of the two-factor model of the B-PBCS.

Note. B-PBCS = Brief Partner Behavioral Control Scale. ${ }^{* * *}$ Standardized factor loadings are significant at the $p<.001$ level.

were .90 and .86 for covert partner behavioral control, .76 and .76 for overt partner behavioral control, and .84 and .82 for total scale in Samples 1 and 2, respectively. Inter-item correlation matrixes revealed that the inter-item correlations were moderate to high in magnitude within factors in both data sets. Corrected-item total correlations ranged from .68 to .78 and .57 to .74 for covert partner behavioral control and ranged from .46 to .60 and .53 to .64 for overt partner behavioral control in Samples 1 and 2, respectively. As a result, all these findings indicate that the B-PBCS has a good level of internal consistency.

Test-retest reliability. In order to assess the test-retest reliability, the B-PBCS was administered to the sample across a 14-week time in- terval. Test-retest reliability coefficients were calculated $.59(p<.01, n=182)$ for covert partner behavioral control, .69 for overt partner behavioral control $(p<.01, n=182)$, and $.63(p<.01, n=182)$ for total scale. The results demonstrated that the B-PBCS scores are stable over time.

\section{Predictive Validity}

Using structural path modeling and data from 182 emerging adults, we examined the predictive role of the B-PBCS measured at Time 1 (baseline assessment) in romantic relationship conflict and psychological distress measured at Time 2 (14-week follow-up assessment). The latent predictor variables were 
covert and overt partner behavioral control, and the latent outcome variables were romantic relationship conflict and psychological distress in the model. The latent variables of covert and overt partner behavioral control were represented by corresponding B-PBCS items. The romantic relationship conflict latent variable was represented by frequency, intensity, and resolution subscales. Psychological distress latent variable was represented by three parcels. Parcels were created using an item-to-construct balance approach (Little, Cunningham, Shahar, \& Widaman, 2002). Sex, age, and relationship duration were used as covariates in the analyses. The measurement model, comprising four latent variables with 15 indicators, indicated a good fit to the data: $\chi^{2}(83)=111.817, p<.05, \chi^{2} / d f=1.35, \mathrm{CFI}=$ $.98, \mathrm{TLI}=.97$, and RMSEA $=.04 \mathrm{Cl}[.02, .06]$. The factor loadings ranged from .57 to .88 for covert partner behavioral control, .64 to .82 for overt partner behavioral control, .67 to .76 for romantic relationship conflict, .90 to .94 for psychological distress, and all were statistically significant $(p<.001)$. The fit indices for structural model showed a good fit to the data as well: $\chi^{2}(118)=204.531, p<.001$, $\chi^{2} / d f=1.73, \mathrm{CFI}=.94, \mathrm{TLI}=.92$, and RMSEA $=.06 \mathrm{Cl}[.05, .08]$. The results of the path analysis are depicted in Figure 2. As expected, overt partner behavioral control at Time 1 had a statistically significant positive effect on romantic relationship conflict $(B=.51$, $p<.001)$ and psychological distress $(B=.22$,

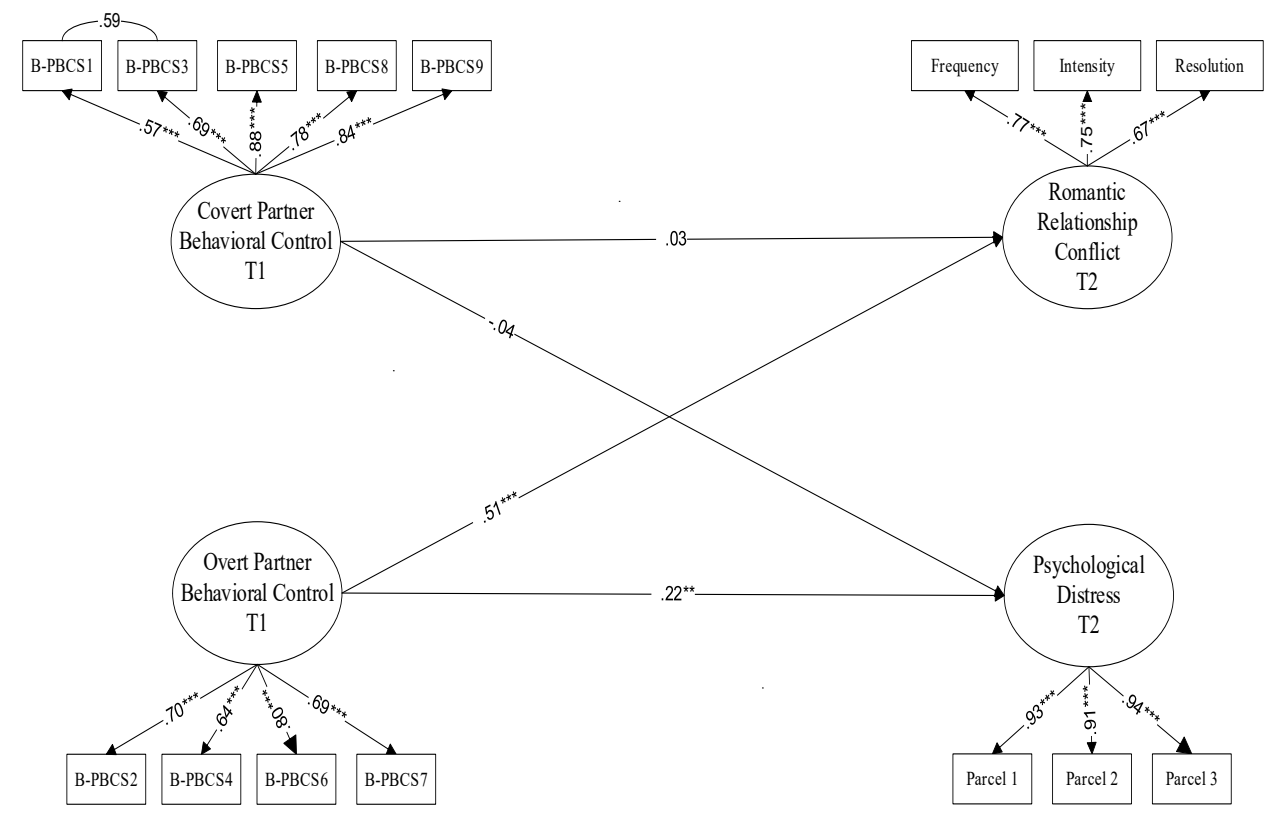

Figure 2 Structural links between the covert partner behavioral control, overt partner behavioral control, romantic relationship conflict, and psychological distress $(n=182)$.

Note. The covariates (gender, age, relationship duration) were included in the analyses but are omitted to enhance clarity of the figure. Standardized regression weights are presented. T1 = Time 1. T2 = Time $2 .{ }^{* *} p<.01,{ }^{* * *} p<.001$ 
$p<.01$ ) at Time 2. Counter to expectations, covert partner behavioral control at Time 1 was not significantly linked with romantic relationship conflict $(\theta=.03, p>.05)$ and psychological distress $(B=-.04, p>.05)$ at Time 2. The squared multiple correlations indicated that the perceived partner behavioral control at Time 1 accounted for $32 \%$ of the variance in romantic relationship conflict and $11 \%$ of the variance in psychological distress at Time 2 in emerging adults. Taken together, the results confirmed the predictive validity of the B-PBCS on individual and relational processes.

\section{Discussion}

The primary purpose of the present study was to develop and validate the psychometric properties of the Brief Partner Behavioral Control Scale (B-PBCS). Results from the research indicate that the $B-P B C S$ is a brief, valid, and reliable measure for assessing partner behavioral control in premarital romantic relationships of emerging adults. The findings obtained from exploratory and confirmatory factor analyses confirmed that the B-PBCS involves two distinct dimensions: overt and covert partner behavioral control. The results of the measurement invariance analyses demonstrate that the factor structure and loadings of the B-PBCS is the same across time. Cronbach's alpha and test-retest reliability indices ensure that the B-PBCS has stable and reliable psychometric properties. The results also confirm the predictive validity of the B-PBCS.

Particularly, in the present research, we found that overt partner behavioral control was a significant predictor of both romantic relationship conflict and psychological distress over time (14-week). Indeed, this finding was consistent with Khaleque's (2012) finding that perceived partner behavioral control associates with lower levels of psychological adjustment. However, unexpectedly, covert partner behavioral control was not a significant predictor of either relationship conflict or psychological distress. Based on relationship maintenance literature, some scholars (Goodboy, Dainton, Borzea, \& Goldman, 2017) emphasize that allowing control could be used as a relationship maintenance strategy to keep the less committed partner in the relationship. As such, allowing covert partner behavioral control serves the function of keeping the relationship in existence. Dainton and Gross (2008) suggest that romantic partners experiencing passionate love are also likely to allow control in order to maintain passion in their relationships. However, Dainton and Gross (2008) also caution that although partners using such strategies meet their personal needs and/or feel better about their relationships, negative maintenance behaviors (e.g., allowing control) involve some level of dysfunction. Moreover, from the perspective of self-determination theory (Deci \& Ryan, 2014), it would also be likely that partners allowing covert behavioral control may be using this relationship maintenance strategy in order to fulfill their basic psychological need satisfaction. Finally, the perception of behavioral control would be seen as a social construction to some degree, and thus it is likely that partners possess distinct interpretations of its nature. Particularly, covert control behaviors including tracking the daily behaviors and routines of the partner are likely to be perceived as a manifestation of interest, warmth, and love by the partner, particularly considering the collectivistic nature of Turkish culture. Indeed, empirical evidence (e.g., Toplu-Demirtaş, Murray, \& HatipoğluSümer, 2019) indicates that aggressive/controlling behaviors are highly prevalent in Turkish emerging adults' dating relationships and they are commonly perceived as an indicator of possessiveness in the romantic relation- 
ships (Toplu-Demirtaş \& Fincham, 2018). Toplu-Demirtaş, Hatipoğlu, and Fincham's (2017) study also supports that abusive behaviors like jealousy (doing something just to make partner jealous) are perceived as a sign of love by Turkish young adults. As a result, the present finding regarding the nonsignificant links between covert partner behavioral control and outcome variables (romantic relationship conflict and psychological distress) could be influenced by individual, relational as well as cultural factors. But, further research is needed to elaborate on this finding.

The present study extends the partner behavioral control research in important ways. Although scholars (Dush \& Amato, 2005; La Guardia \& Patrick, 2008; Patrick et al., 2007; Sağkal \& Özdemir, 2020) have endorsed the significance of romantic interactions on individual and relational outcomes, limited research has paid attention to the construct of partner behavioral control in the close relationships literature. In addition, the existing measures (e.g., Fincham et al., 2008; Graham-Kevan \& Archer, 2005; Rohner, 2005b) either mostly addressed overt partner behavioral control or targeted coercive behaviors. However, to the best of our knowledge, no research to date has captured the covert control dimension in the assessment of partner behavioral control. Thus, the present study, offering a brief and comprehensive measure of partner behavioral control, provides a significant step in addressing this gap in the literature. In addition, the B-PBCS, providing a framework for identifying patterns of partner behavioral control, hopefully inspires future research in the close relationships field.

The results of this study can also help counselors and therapists to improve their assessment and treatment protocols in the helping process. Based on Rigazio-DiGilio, Ivey, Kunkler-Peck, and Grady's (2005) descriptions, the B-PBCS including the features of having emphasis on perceived behaviors, subjective experiences, unrecognized topics, and multiple perspectives seems to meet several criteria needed to be identified as a measure best adapted for clinical use. The B-PBCS as a self-report survey assesses the extent to which partners are perceived to place overt and covert limits, restrictions, proscriptions, and/or prescriptions on behaviors. By using the B-PBCS, therapists could help couples to examine the quality of relationships they currently experience with one another. The B-PBCS would also help couples to explore the effects of perceived partner behavioral control on the intrapsychic and interpersonal problems that led them to seek a professional treatment. According to Rigazio-Digilio and Rohner (2015), with the use of microcounseling skills, therapists could invite partners to understand their subjective perceptions and experiences regarding partner behavioral control, determine therapeutic goals, and establish a collaborative relationship for a therapeutic change. Based on Rigazio-Digilio and Rohner's explanations, we recommend therapists to use the B-PBCS as a brief and psychometrically sound assessment tool in therapy sessions and interpret the results interactively with their clients. We believe that using such an interactive and collaborative clinical application of the B-PBCS promises to increase therapeutic alliance and shared responsibility between therapists and their clients and to promote treatment processes and outcomes in therapeutic interventions.

The present study has some limitations that need to be considered when interpreting the findings as well. First of all, the psychometric properties of the B-PBCS were tested with emerging adults living in a western city of Turkey. Further research is needed to validate the scale across different regions of Turkey. Moreover, in order to ensure the generalizability of the findings, researchers are encour- 
aged to validate the B-PBCS across cultures in future studies. Secondly, in this study, we assessed the psychometric properties of the $\mathrm{B}-\mathrm{PBCS}$ in premarital romantic relationships of emerging adults. But, partner behavioral control is also experienced in all relationship types. Therefore, future studies would be well-served to test the psychometric properties of the B-PBCS across different relationship types, such as cohabiting and/or marital relationships. Thirdly, as the data were not dyadic, we only tried to understand the actor effects of partner behavioral control on relationship conflict and psychological distress. By using a dyadic research design, future studies could also aim to explore the cross-partner effects. Furthermore, future researchers could also longitudinally investigate the antecedents and outcomes of partner behavioral control. Finally, as the sample comprised a disproportionate number of females, further research is needed to conduct measurement invariance of the B-PBCS across gender.

In conclusion, the present study is the first to validate a brief and comprehensive measure of partner behavioral control. The empirical findings obtained from exploratory and confirmatory factor analyses, measurement invariance tests, Cronbach's alpha and test-retest reliability analyses as well as predictive validity study highlight that B-PBCS is a brief, valid, and reliable measure of partner behavioral control. These results suggest that researchers and practitioners can validly and reliably utilize the B-PBCS in assessing perceived partner behavioral control in premarital romantic relationships of emerging adults. The present study provides an avenue for research, training, and practice. But, further exploration of the construct of partner behavioral control is also needed to make greater contribution to the field of close relationships.

\author{
Authors' ORCID \\ Ali Serdar Sağkal \\ https://orcid.org/0000-0002-2597-8115 \\ Yalçın Özdemir \\ https://orcid.org/0000-0003-2698-0900 \\ Didem Aydoğan \\ https://orcid.org/0000-0002-7163-3003
}

\section{References}

Arnett, J. J. (2000). Emerging adulthood: A theory of development from the late teens through the twenties. American Psychologist, 55(5), 469-480. https://doi.org/10.1037/0003-066X.55.5.469

Arnett, J. J. (2004). Emerging adulthood: The winding road from the late teens through the twenties. New York, NY: Oxford University Press.

Arnett, J. J. (2007). Emerging adulthood: What is it, and what is it good for? Child Development Perspectives, 1(2), 68-73. https://doi.org/10.1111/ j.1750-8606.2007.00016.x

Arroyo, K., Lundahl, B., Butters, R., Vanderloo, M., \& Wood, D. S. (2017). Short-term interventions for survivors of intimate partner violence: A systematic review and meta-analysis. Trauma, Violence, \& Abuse, 18(2), 155-171. https://doi. org $/ 10.1177 / 1524838015602736$

Barber, B. K., Olsen, J. H., \& Shagle, S. C. (1994). Associations between parental psychological and behavioral control and youth internalized and externalized behaviors. Child Development, 65(4), 1120-1136. https://doi.org/10.1111/i.1467-8624.1994. tb00807.x

Barry, C. M., Madsen, S. D., Nelson, L. J., Carroll, J. S., \& Badger, S. (2009). Friendship and romantic relationship qualities in emerging adulthood: Differential associations with identity development and achieved adulthood criteria. Journal of Adult Development, 16(4), 209-222. https:// doi.org/10.1007/s10804-009-9067-x

Basha, S. E. (2014). Perceived intimate partner rejection, behavioral control and it's relation to personality dispositions: Resilience as a protective factor among married adults in Egypt. Indian Journal of Health and Wellbeing, 5(11), 1255-1262. 
Belu, C. F., Lee, B. H., \& O’Sullivan, L. F. (2016). It hurts to let you go: Characteristics of romantic relationships, breakups and the aftermath among emerging adults. Journal of Relationships Research, 7, 1-11. https://doi.org/10.1017/jrr.2016.11

Byrne, B. M. (2010). Structural equation modeling with AMOS: Basic concepts, applications, and programming ( $2^{\text {nd }}$ ed.). New York, NY: Routledge.

Centers for Disease Control and Prevention. (2018). Intimate partner violence. Retrieved from https:// www.cdc.gov/violenceprevention/intimatepartnerviolence/

Dainton, M., \& Gross, J. (2008). The use of negative behaviors to maintain relationships. Communication Research Reports, 25(3), 179-191. https://doi.org/10.1080/08824090802237600

Deci, E. L., \& Ryan, R. M. (2014). Autonomy and need satisfaction in close relationships: Relationships motivation theory. In N. Weinstein (Ed.), Human motivation and interpersonal relationships: Theory, research, and applications ( $\mathrm{pp}$. 53-73). Dordrecht: Springer.

DiBello, A. M., Preddy, T. M., Øverup, C. S., \& Neighbors, C. (2017). Understanding the context of romantic partner relational victimization: Links between relationship satisfaction, depressive symptoms, and alcohol-related problems. Psychology of Violence, 7(4), 543-552. https://doi. org/10.1037/vio0000064

Dush, C. M. K., \& Amato, P. R. (2005). Consequences of relationship status and quality for subjective well-being. Journal of Social and Personal Relationships, 22(5), 607-627. https://doi. org/10.1177/0265407505056438

Eurostat (2019). Marriage and divorce statistics. Retrieved from https://ec.europa.eu/eurostat/ statisticsexplained/index.php/Marriage and divorce statistics

Fanslow, J. L., \& Robinson, E. M. (2011). Sticks, stones, or words? Counting the prevalence of different types of intimate partner violence reported by New Zealand women. Journal of Aggression, Maltreatment \& Trauma, 20(7), 741-759. https://doi.org/1 $\underline{0.1080 / 10926771.2011 .608221}$

Fincham, F. D., \& Cui, M. (2011). Emerging adulthood and romantic relationships: An introduction. In F. D. Fincham \& M. Cui (Eds.), Romantic relationships in emerging adulthood (pp. 1-12). New York, NY: Cambridge University Press.
Fincham, F. D., Cui, M., Braithwaite, S., \& Pasley, K. (2008). Attitudes toward intimate partner violence in dating relationships. Psychological Assessment, 20(3), 260-269. https://doi. org/10.1037/1040-3590.20.3.260

Fine, M. A., \& Harvey, J. H. (Eds.). (2006). Handbook of divorce and relationship dissolution. New York, NY: Routledge.

Follingstad, D. R., Bradley, R. G., Helff, C. M., \& Laughlin, J. E. (2002). A model for predicting dating violence: Anxious attachment, angry temperament, and need for relationship control. Violence and Victims, 17(1), 35-47. doi: 10.1891/ vivi.17.1.35.33639

Fox, J., \& Tokunaga, R. S. (2015). Romantic partner monitoring after breakups: Attachment, dependence, distress, and post-dissolution online surveillance via social networking sites. Cyberpsychology, Behavior, and Social Networking, 18(9), 49-498. https://doi.org/10.1089/cyber.2015.0123

Garcia-Moreno, C., Pallitto, C., Devries, K., Stockl, H., Watts, C., \& Abrahams, N. (2013). Global and regional estimates of violence against women: Prevalence and health effects of intimate partner violence and non-partner sexual violence. Geneva, Switzerland: World Health Organization.

Geitsidou, A., \& Giovazolias, T. (2016). Intimate partner acceptance-rejection and subjective well-being: What is the role of resilience? Journal of Child and Family Studies, 25(11), 3260-3269. https://doi.org/10.1007/s10826-016-0493-7

Goodboy, A. K., Dainton, M., Borzea, D., \& Goldman, Z. W. (2017). Attachment and negative relational maintenance: Dyadic comparisons using an actor-partner interdependence model. Western Journal of Communication, 81(5), 541-559. https://doi. org/10.1080/10570314.2017.1302601

Graham-Kevan, N., \& Archer, J. (2005). Investigating three explanations of women's relationship aggression. Psychology of Women Quarterly, 29(3), 270-277. https://doi.org/10.1111/i.14716402.2005.00221.x

Graham-Kevan, N., \& Archer, J. (2008). Does controlling behavior predict physical aggression and violence to partners? Journal of Family Violence, 23, 539-548. https://doi.org/10.1007/s10896-0089162-y

Grych, J. H., Seid, M., \& Fincham, F. D. (1992). Assessing marital conflict from the child's perspec- 
tive: The Children's Perception of Interparental Conflict Scale. Child Development, 63(3), 558572. https://doi.org/10.2307/1131346

Kessler, R. C., Andrews, G., Colpe, L. J., Hiripi, E., Mroczek, D. K., Normand, S. L., ... \& Zaslavsky, A. M. (2002). Short screening scales to monitor population prevalences and trends in non-specific psychological distress. Psychological Medicine, 32(6), 959-976. https://doi.org/10.1017/ $\underline{\mathrm{S} 0033291702006074}$

Khaleque, A. (2001). Parental acceptance-rejection, psychological adjustment, and intimate adult relationships (Unpublished master's thesis). University of Connecticut, Storrs.

Khaleque, A. (2012). Adult intimate relationships and psychological adjustment. Psychological Studies, 57(1), 95-100. https://doi.org/10.1007/ s12646-011-0134-6

Khaleque, A., \& Ali, S. (2017). A systematic review of meta-analyses of research on interpersonal acceptance-rejection theory: Constructs and measures. Journal of Family Theory \& Review, 9(4), 441-458. https://doi.org/10.1111/ iftr.12228

Khaleque, A., \& Rohner, R. P. (2013). Effects of multiple acceptance and rejection on adults' psychological adjustment: A pancultural study. Social Indicators Research, 113(1), 393-399. https:// doi.org/10.1007/s11205-012-0100-2

Khaleque, A., Rohner, R. P., \& Laukkala H. (2008). Intimate partner acceptance, parental acceptance, behavioral control, and psychological adjustment among Finnish adults in ongoing 119 attachment relationships. Cross-Cultural Research, 42(1), 3545. https://doi.org/10.1177/1069397107309755

Khaleque, A., Shirin, A., \& Uddin, M. K. (2013). Attachment relationships and psychological adjustment of married adults. Social Indicators Research, 10(1), 237-244. doi: 10.1007/sl 1205011-9926-2

La Guardia, J. G., \& Patrick, H. (2008). Self-determination theory as a fundamental theory of close relationships. Canadian Psychology, 49(3), 201209. https://doi.org/10.1037/a0012760

Little, T. D., Cunningham, W. A., Shahar, G., \& Widaman, K. F. (2002). To parcel or not to parcel: Exploring the question, weighing the merits. Structural Equation Modeling, 9(2), 151-173. https:// doi.org/10.1207/S15328007SEM0902 1
Lövestad, S., Löve, J., Vaez, M., \& Krantz, G. (2017). Prevalence of intimate partner violence and its association with symptoms of depression; a cross-sectional study based on a female population sample in Sweden. BMC Public Health, 17(1), 335. https://doi.org/10.1186/s12889-017-4222-y

Mackinnon, S. P., Sherry, S. B., Antony, M. M., Stewart, S. H., Sherry, D. L., \& Hartling, N. (2012). Caught in a bad romance: Perfectionism, conflict, and depression in romantic relationships. Journal of Family Psychology, 26(2), 215225. https://doi.org/10.1037/a0027402

Malik, F., \& Rohner, R. P. (2016). Spousal rejection as a risk factor for parental rejection of children among Pakistani families in the US. Journal of Family Violence, 31(1), 119-125. https://doi. org/10.1007/s10896-015-9756-0

Mayseless, O., \& Keren, E. (2014). Finding a meaningful life as a developmental task in emerging adulthood: The domains of love and work across cultures. Emerging Adulthood, 2(1), 63-73. https://doi.org/10.1177/2167696813515446

Murphy, C. M., \& Hoover, S. A. (1999). Measuring emotional abuse in dating relationships as a multifactorial construct. Violence and Victims, 14(1), 39-53.

Parmar, P., \& Rohner, R. P. (2005). Relations among perceived intimate partner acceptance, remembered parental acceptance, and psychological adjustment among young adults in India. Ethos, 33(3), 402-413.

Patrick, H., Knee, C. R., Canevello, A., \& Lonsbary, C. (2007). The role of need fulfillment in relationship functioning and well-being: A self-determination theory perspective. Journal of Personality and Social Psychology, 92(3), 434-457.

Rigazio-DiGilio, S. A., Ivey, A. E., Kunkler-Peck, K. P., \& Grady, L T. (2005). Community genograms: Using individual, family and cultural narratives with clients. New York, NY: Teacher's College Press, Columbia University.

Rigazio-DiGilio, S. A., \& Rohner, R. P. (2015). Interpersonal acceptance-rejection theory and clinical applications. In E. Kourkoutas, A. Hart, \& A. Mouzaki (Eds.), Innovative practices and interventions for children and adolescents with psychosocial difficulties and disorders (pp. 476515). Newcastle Upon Tyne, UK: Cambridge Scholars Publications. 
Rohner, R. P. (2005a). Child Parental AcceptanceRejection/Control Questionnaire (PARQ/Control) test manual. In R. P. Rohner \& A. Khaleque (Eds.), Handbook for the study of parental acceptance and rejection ( ${ }^{\text {th }}$ ed., pp. 137-186). Storrs, CT: Rohner Research Publications.

Rohner, R. P. (2005b). Intimate Partner Acceptance-Rejection/ Control Questionnaire (IPAR/ CQ) test manual. In R. P. Rohner \& A. Khaleque (Eds.), Handbook for the study of parental acceptance and rejection ( $4^{\text {th }}$ ed., pp. 227-242). Storrs, CT: Rohner Research Publications.

Rohner, R. P. (2014). PARTheory gets a new name. Interpersonal Acceptance, 8(3), 6.

Rohner, R. P. (2016). Introduction to Interpersonal Acceptance-Rejection Theory (IPARTheory) and evidence. Online Readings in Psychology and Culture, 6(1), 1-40. https://doi.org/10.9707/2307$\underline{0919.1055}$

Rohner, R. P., \& Khaleque A. (2008). Relations between perceived partner and parental acceptance, behavioral control, and psychological adjustment among heterosexual adult women in the U.S. In F. Columbus (Ed.), Family relations: Behavioral, psychological, and sociological as pect (pp. 187-197). New York, NY: Noca Science Publishers, Inc.

Rohner, R. P., \& Khaleque, A. (2010). Testing central postulates of Parental Acceptance-Rejection Theory (PARTheory): A meta-analysis of cross-cultural studies. Journal of Family Theory \& Review, 2(1), 73-87. https://doi.org/10.1111/ j.1756-2589.2010.00040.x

Rohner, R. P., Melendez, T., \& Kraimer-Rickaby, L. (2008). Intimate partner acceptance, parental acceptance in childhood, and psychological adjustment among American adults in ongoing attachment relationships. Cross-Cultural Research, 42(1), 1322. https://doi.org/10.1177/1069397107309750

Sağkal, A. S., \& Özdemir, Y. (2017, October). Turkish adaptation of the Kessler Psychological Distress Scale (K10). Paper presented at the meeting of II. International Congress of Youth Researches, Muğla.

Sağkal, A. S., \& Özdemir, Y. (2019). Interparental conflict and emerging adults' couple satisfaction: The mediating roles of romantic relationship conflict and marital attitudes. Başkent University Journal of Education, 6(2), 181-191.
Sağkal, A. S., \& Özdemir, Y. (2020). I am satisfied with my sweetheart, therefore I am satisfied with my life (and vice versa): A cross-lagged autoregressive panel model. The Journal of General Psychology, 147(4), 381-397. https://doi.org/ 10.1080/00221309.2019.1688757

Sağkal, A. S., Özdemir, Y., \& Koruklu, N. (2018). Direct and indirect effects of father-daughter relationship on adolescent girls' psychological outcomes: The role of basic psychological need satisfaction. Journal of Adolescence, 68, 32-39. https:// doi.org/10.1016/j.adolescence.2018.07.001

Shek, D. T. L. (2006). Perceived parental behavioral control and psychological control in Chinese adolescents in Hong Kong. The American Journal of Family Therapy, 34(2), 163-176. https://doi. org/10.1080/01926180500357891

Sleath, E., Walker, K., \& Tramontano, C. (2018). Factor structure and validation of the Controlling Behaviors Scale-Revised and Revised Conflict Tactics scale. Journal of Family Issues, 39(7), 1880-1903. https://doi.org/10.1177/0192513X17729721

Snyder, A. R. (2006). Risky and casual sexual relationships among teens. In A. C. Crouter \& A. Booth (Eds.), Romance and sex in adolescence and emerging adulthood: Risk and opportunities (pp. 161-169). Mahwah, NJ: Lawrence Erlbaum Associates.

Stark, E. (2007). Coercive control: How men entrap women in personal life. New York, NY: Oxford University Press.

Steinberg, L., Elmen, J. D., \& Mounts, N. S. (1989). Authoritative parenting, psychosocial maturity, and academic success among adolescents. Child Development, 60(6), 1424-1436. https://doi. org/10.2307/1130932

Straus, M. A., Hamby, S. L., Boney-McCoy, S., \& Sugarman, D. B. (1996). The Revised Conflict Tactics Scale (CTS2): Development and preliminary psychometric data. Journal of Family Issues, 17(3), 283-316. https://doi.org/10.1177/019251396017003001

Tabachnick, B. G., \& Fidell, L. S. (2007). Using multivariate statistics ( $5^{\text {th }}$ ed.). Boston, MA: Allyn \& Bacon.

Toplu-Demirtaş, E., \& Fincham, F. D. (2018). Dating infidelity in Turkish couples: The role of attitudes and Intentions. The Journal of Sex Research, 55(2), 252-262. https://doi.org/10.1080 /00224499.2017.1365110 
Toplu-Demirtaş, E. T., Hatipoğlu-Sümer, Z., \& Fincham, F. D. (2017). Intimate partner violence in Turkey: The Turkish Intimate Partner Violence Attitude Scale-Revised. Journal of Family Violence, 32(3), 349-356. https://doi.org/10.1007/ s10896-016-9852-9

Toplu-Demirtaş, E., Murray, C., \& Hatipoğlu-Sümer, Z. (2019). Attachment insecurity and restrictive engulfment in college student relationships: The mediating role of relationship satisfaction. Journal of Aggression, Conflict and Peace Research, 11(1), 2437. https://doi.org/10.1108/JACPR-11-2017-0333

Ulu, P., \& Fişıloğlu, H. (2004). Çocukların Evlilik Çatışmasını Algılaması Ölçeği'nin geçerlik ve güvenirlik çalışması [A validity and reliability study of the Children's Perceptions of Interparental Conflict Scale]. Türk Psikoloji Yazıları, 7(14), 61-75.
Van Ouytsel, J., Walrave, M., Ponnet, K., Willems, A. S., \& Van Dam, M. (2019). Adolescents' perceptions of digital media's potential to elicit jealousy, conflict and monitoring behaviors within romantic relationships. Cyberpsychology: Journal of Psychosocial Research on Cyberpspace, 13(3), 1-21. https://doi.org/10.5817/CP2019-3-3

Walker, K., Sleath, E., \& Tramontano, C. (2021). The prevalence and typologies of controlling behaviors in a general population sample. Journal of Interpersonal Violence, 6(1-2), NP474-NP503. https://doi.org/10.1177/0886260517731785

Worthington, R. L., \& Whittaker, T. A. (2006). Scale development research: A content analysis and recommendations for best practices. The Counseling Psychologist, 34(6), 806-838. https://doi. org/10.1177/0011000006288127 